\title{
POINCARÉ SERIES OF EMBEDDED FILTRATIONS
}

\author{
ANN LEMAHIEU
}

\begin{abstract}
In this article, we define a Poincaré series on a subspace of a complex analytic germ, induced by a multi-index filtration on the ambient space. This Poincaré series differs from Poincaré series studied before in the sense that there is no notion of fibre that corresponds to our Poincaré series. We compute this Poincaré series for subspaces defined by principal ideals. For plane curve singularities and nondegenerate singularities this Poincaré series yields topological and geometric information. We compare this Poincaré series with the one introduced in [E,G-Z2]. In few cases, they are equal and we show that the Poincaré series we consider in this paper in general yields more information.
\end{abstract}

\section{Introduction}

In $[\mathrm{C}, \mathrm{D}, \mathrm{K}]$, one introduced a Poincaré series induced by a filtration on the ring of germs of a complex variety. This Poincaré series has been studied for several kinds of singularities, see for example [C,D,G-Z1], [C,D,G-Z2], [C,D,G-Z3], [E,G-Z1], [C,H,R], $[\mathrm{L}],[\mathrm{GP}, \mathrm{H}]$ and $[\mathrm{N}]$. In some cases, this Poincaré series determines the topology of the singularity and is related to its zeta function of monodromy.

In these works, one considers multi-index filtrations defined by valuations on the local ring at the singularity and in [E,G-Z1] and recently in [E,G-Z2] one considers valuations on an ambient smooth space of the singularity that correspond to facets of the Newton polyhedron. Here, we study Poincaré series induced by multi-index filtrations coming from arbitrary valuations on the ambient space where at least one of them is centred at the maximal ideal of the local ring of the singularity considered in the ambient space. In an upcoming paper, we study this Poincaré series also for valuations where none of them is centred at the maximal ideal.

The Poincaré series we introduce here is defined in an algebraic way and differs from Poincaré series studied before in the sense that there is no notion of fibre that corresponds to our Poincaré series. We go into this in Section 1. We compute this Poincaré series for a subspace corresponding to a principal ideal. A nice A'Campo type formula shows up. In Section 2, we compare our Poincaré series with the one defined in [E,G-Z2]. The Poincaré series in [E,G-Z2] is consistent with a notion of geometric fibre but the price to pay is that this filtration is less richer than the one we consider here. This follows also from the formulas they computed for their Poincaré series. When the Newton polytope is bi-stellar, we show that both Poincaré series coincide. The property of bi-stellar generalizes the notion of stellar introduced in [E,G-Z2]. In Section 3, we study this Poincaré series for a plane curve singularity that is a general element in an ideal in $\mathbb{C}\{x, y\}$ where the embedded filtration comes from the Rees valuations of the ideal. Using the result in [C,D,G-Z4], we show that the Poincaré series determines and is determined by the embedded topology of the plane

Received by the editors September 27, 2010. Revision received April 23, 2011. 
curve singularity. In Section 4, we compare different Poincaré series for affine toric varieties. In Section 5, we study the Poincaré series for nondegenerate singularities where the embedded filtration is now induced from the Newton polyhedron. We show that one can recover the Newton polyhedron from the Poincaré series and thus, in particular, that the zeta function of monodromy can be deduced from the Poincaré series.

\section{Poincaré series associated to embedded filtrations}

Let $(X, o)$ be a germ of a complex analytic space and let $\mathcal{O}_{X, o}$ be the local ring of germs of functions on $(X, o)$. Let $\underline{\nu}=\left\{\nu_{1}, \ldots, \nu_{r}\right\}$ be a set of order functions from $\mathcal{O}_{X, o}$ to $\mathbb{Z} \cup\{\infty\}$, i.e. functions $\nu_{j}$ that satisfy $\nu_{j}(f+g) \geq \min \left\{\nu_{j}(f), \nu_{j}(g)\right\}$ and $\nu_{j}(f g) \geq \nu_{j}(f)$, for all $f, g \in \mathcal{O}_{X, o}, 1 \leq j \leq r$. An order function $\nu_{j}$ is called a valuation if moreover it satisfies $\nu_{j}(f g)=\nu_{j}(f)+\nu_{j}(g)$, for all $f, g \in \mathcal{O}_{X, o}$. In particular, when $(X, o)$ is irreducible, $\underline{\nu}$ can be a set of discrete valuations of the function field $\mathbb{C}(X)$ whose valuation rings contain $\mathcal{O}_{X, o}$. The set $\underline{\nu}$ defines a multiindex filtration on $\mathcal{O}_{X, o}$ by the ideals

$$
M(\underline{v}):=\left\{g \in \mathcal{O}_{X, o} \mid \nu_{j}(g) \geq v_{j}, 1 \leq j \leq r\right\}, \quad \underline{v} \in \mathbb{Z}^{r} .
$$

If the dimensions of the complex vector spaces $M(\underline{v}) / M(\underline{v}+\underline{1})$ are finite for all $\underline{v} \in \mathbb{Z}^{r}$, then originally (see $[\mathrm{C}, \mathrm{D}, \mathrm{K}]$ and $[\mathrm{C}, \mathrm{D}, \mathrm{G}-\mathrm{Z} 2]$ ) the Poincaré series associated to this multi-index filtration was defined as

$$
P \frac{\nu}{X}\left(t_{1}, \ldots, t_{r}\right):=\frac{\prod_{j=1}^{r}\left(t_{j}-1\right)}{\left(t_{1} \cdots t_{r}-1\right)} \sum_{\underline{v} \in \mathbb{Z}^{r}} \operatorname{dim}(M(\underline{v}) / M(\underline{v}+\underline{1})) \underline{t}^{\underline{v}} .
$$

Let us now consider an ideal $\mathcal{I}$ in $\mathcal{O}_{X, o}$. We will define a Poincaré series associated to an embedded filtration on $\mathcal{O}_{X, o} / \mathcal{I}$. Let $V$ be the analytic subspace of $X$ determined by the ideal $\mathcal{I}$ and let the map $\varphi: \mathcal{O}_{X, o} \rightarrow \mathcal{O}_{X, o} / \mathcal{I}$ define the embedding of $V$ in $X$. We set $I(\underline{v}):=\varphi(M(\underline{v})+\mathcal{I})$. In general, the ideals $I(\underline{v})$ define a multi-index filtration on $\mathcal{O}_{V, o}$ which is not induced by a set of order functions on $V$, i.e., there do not have to exist order functions $\mu_{1}, \ldots, \mu_{r}$ on $V$ such that $I(\underline{v})=\left\{g \in \mathcal{O}_{V, o} \mid \mu_{j}(g) \geq v_{j}, 1 \leq\right.$ $j \leq r\}$. The existence of such functions for $\{I(\underline{v})\}$ is equivalent with the condition that

$$
I\left(\underline{v_{1}}\right) \cap I\left(\underline{v_{2}}\right)=I(\underline{v}),
$$

where $v_{1}$ and $v_{2}$ are arbitrary tuples in $\mathbb{Z}^{r}$ and $\underline{v}$ is the tuple of the componentwise maxima of $v_{1}$ and $\underline{v_{2}}$. Indeed, if Condition (1.1) holds, then one has order functions $\mu_{j}$ on $V$ with $\mu_{j}(g)=v_{j}$ if and only if $g \in I\left(0, \ldots, 0, v_{j}, 0, \ldots, 0\right) \backslash I\left(0, \ldots, 0, v_{j}+\right.$ $1,0, \ldots, 0)$.

Definition 1.1. The Poincaré series associated to the embedded multi-index filtration given by the ideals $I(\underline{v})$ is the series

$$
\mathcal{P} \frac{\nu}{V}\left(t_{1}, \ldots, t_{r}\right):=\frac{\prod_{j=1}^{r}\left(t_{j}-1\right)}{\left(t_{1} \cdots t_{r}-1\right)} \sum_{\underline{v} \in \mathbb{Z}^{r}} \operatorname{dim}(I(\underline{v}) / I(\underline{v}+\underline{1})) \underline{t}^{\underline{v}} .
$$

It is useful to notice that $\operatorname{dim}(I(\underline{v}) / I(\underline{v}+\underline{1}))=\operatorname{dim}(J(\underline{v}) / J(\underline{v}+\underline{1}))$, where $J(\underline{v}):=$ $M(\underline{v})+\mathcal{I}$. If $\{I(\underline{v})\}$ is defined by order functions $\mu_{1}, \ldots, \mu_{r}$, then one has a notion 
of fibre because to every $g \in \mathcal{O}_{V, o}$ can attach a value $\underline{\mu}(g)=\left(\mu_{1}(g), \ldots, \mu_{r}(g)\right)$. Concretely, consider the projection map

$$
\begin{aligned}
j_{\underline{v}}: I(\underline{v}) & \longrightarrow D_{1}(\underline{v}) \times \cdots \times D_{r}(\underline{v}) \\
g & \longmapsto\left(a_{1}(g), \ldots, a_{r}(g)\right),
\end{aligned}
$$

where for $1 \leq j \leq r$ the space $D_{j}(\underline{v})=I(\underline{v}) / I\left(\underline{v}+e_{j}\right)$ where $e_{j}$ is the $r$-tuple with $j$ th component equal to 1 and the other components equal to 0 , and $a_{j}(g)$ is the class of $g$ in $D_{j}(\underline{v}), 1 \leq j \leq r$. As Condition (1.1) then holds, the following expressions

$$
\begin{aligned}
& F_{\underline{v}}:=\operatorname{Im} j_{\underline{v}} \cap\left(D_{1}^{*}(\underline{v}) \times \cdots \times D_{r}^{*}(\underline{v})\right), \\
& F_{\underline{v}}:=(I(\underline{v}) / I(\underline{v+1})) \backslash \bigcup_{i=1}^{r}\left(I\left(\underline{v+e_{i}}\right) / I(\underline{v+1})\right)
\end{aligned}
$$

coincide and one has that $\underline{\mu}(g)=\underline{v}$ if and only if $g+I(\underline{v+1}) \in F_{\underline{v}}$. The space $F_{\underline{v}}$ is invariant with respect to multiplication by nonzero constants; let $\mathbb{P} F_{\underline{v}}=F_{\underline{v}} / \mathbb{C}^{*}$ be the projectivization of $F_{\underline{v}}$. We then have

$$
\mathcal{P} \frac{\nu}{V}(\underline{t})=\sum_{\underline{v} \in \mathbb{Z}^{r}} \chi\left(\mathbb{P} F_{\underline{v}}\right) \underline{t} \underline{\underline{v}},
$$

where $\chi(\cdot)$ denotes the Euler characteristic. If such functions $\mu_{1}, \ldots, \mu_{r}$ do not exist for $\{I(\underline{v})\}$, then (1.2) and (1.3) are possible generalizations of the notion of fibre but we then lose some geometric meaning.

In this paper we will investigate the Poincaré series as in Definition 1.1 and we will see in Example 1.1 that this Poincaré series is different from the two possible ones induced by the "fibres" (1.2) and (1.3).

We now compute this Poincaré series for $\mathcal{I}=(h)$ a principal ideal.

Theorem 1.1. Let $(X, o)$ be irreducible, $\mathcal{I}=(h)$ a principal ideal in $\mathcal{O}_{X, o}$ and $V$ the analytic subspace of $(X, o)$ determined by the ideal $\mathcal{I}$. Let $\underline{\nu}=\left\{\nu_{1}, \ldots, \nu_{r}\right\}$ be a set of discrete valuations of $\mathbb{C}(X)$ centred at the maximal ideal of $\mathcal{O}_{X, o}$. We write $\underline{q}=\underline{\nu}(h)$. Then

$$
\mathcal{P} \frac{\nu}{V}(\underline{t})=(1-\underline{t} \underline{q}) P_{X}^{\frac{\nu}{X}}(\underline{t})
$$

Proof. For a set $A \subset\{1, \ldots, r\}$, let $\underline{1}_{A}$ be the $r$-tuple with $j$ th component equal to 1 if $j \in A$ and else equal to $0,1 \leq j \leq r$. The coefficient of $\underline{t}^{\underline{v}}$ in the left-hand side of (1.4) is

$$
(-1)^{r+1} \sum_{A \subset\{1, \ldots, r\}}(-1)^{\# A} \operatorname{dim} \frac{\mathcal{O}_{X, o}}{J\left(\underline{v}-\underline{1}_{A}+\underline{1}\right)} .
$$

The coefficient of $\underline{t}^{\underline{v}}$ in the right-hand side of (1.4) is equal to

$$
\begin{aligned}
& (-1)^{r+1} \sum_{A \subset\{1, \ldots, r\}}(-1)^{\# A} \operatorname{dim} \frac{\mathcal{O}_{X, o}}{M\left(\underline{v}-\underline{1}_{A}+\underline{1}\right)} \\
& -(-1)^{r+1} \sum_{A \subset\{1, \ldots, r\}}(-1)^{\# A} \operatorname{dim} \frac{\mathcal{O}_{X, o}}{M\left(\underline{v}-\underline{1}_{A}-\underline{q}+\underline{1}\right)} .
\end{aligned}
$$


These sums can be rewritten such that for $A \subset\{1, \ldots, r\}$, it is enough to prove for some $j \in A$ that

$$
\operatorname{dim} \frac{J\left(\underline{v}-\underline{1}_{A}+\underline{1}\right)}{J\left(\underline{v}-\underline{1}_{A \backslash\{j\}}+\underline{1}\right)}=\operatorname{dim} \frac{M\left(\underline{v}-\underline{1}_{A}+\underline{1}\right)}{M\left(\underline{v}-\underline{1}_{A \backslash\{j\}}+\underline{1}\right)}-\operatorname{dim} \frac{M\left(\underline{v}-\underline{1}_{A}-\underline{q}+\underline{1}\right)}{M\left(\underline{v}-\underline{1}_{A \backslash\{j\}}-\underline{q}+\underline{1}\right)} .
$$

This follows immediately from the fact that the kernel of the projection map:

$$
\frac{M\left(\underline{v}-\underline{1}_{A}+\underline{1}\right)}{M\left(\underline{v}-\underline{1}_{A \backslash\{j\}}+\underline{1}\right)} \longrightarrow \frac{J\left(\underline{v}-\underline{1}_{A}+\underline{1}\right)}{J\left(\underline{v}-\underline{1}_{A \backslash\{j\}}+\underline{1}\right)}
$$

is equal to

$$
\frac{(h) M\left(\underline{v}-\underline{1}_{A}-\underline{q}+\underline{1}\right)}{(h) M\left(\underline{v}-\underline{1}_{A \backslash\{j\}}-\underline{q}+\underline{1}\right)} .
$$

Example 1.1. Let $X=\mathbb{C}^{2}$ and $h(x, y)=x^{6} y^{2}+y^{8}$. We choose monomial valuations $\nu_{1}$ and $\nu_{2}$ on $\mathcal{O}_{X, o}$ given by $\nu_{1}\left(x^{a} y^{b}\right)=2 a+3 b$ and $\nu_{2}\left(x^{a} y^{b}\right)=4 a+3 b$. One can compute that the coefficient of $t_{1}^{20} t_{2}^{28}$ in $(1-\underline{t} \underline{q}) P_{X}^{\underline{\nu}}(\underline{t})$ is 0 although both fibres $F_{(20,28)}$ as defined in (1.2) and (1.3) contain the monomial $x^{4} y^{4}$.

\section{Geometric embedded filtrations versus algebraic embedded filtrations.}

Let $h:\left(\mathbb{C}^{d}, o\right) \rightarrow(\mathbb{C}, o)$ be a germ of a holomorphic function. Recently in [E,G-Z2] one considered a Poincaré series on $\mathcal{O}_{V, o}=\mathcal{O}_{\mathbb{C}^{d}, o} /(h)$, induced by a Newton filtration. We write $h(\underline{x})=\sum_{\underline{k} \in \mathbb{Z}_{\geq 0}^{d}} a_{\underline{k}} \underline{x} \underline{k}$, where $\underline{k}=\left(k_{1}, \ldots, k_{d}\right)$ and $\underline{x} \underline{k}=x_{1}^{k_{1}} \cdots x_{d}^{k_{d}}$. The support of $h$ is $\operatorname{supp} h:=\left\{\underline{k} \in \mathbb{Z}_{\geq 0}^{d} \mid a_{\underline{k}} \neq 0\right\}$. The Newton polyhedron of $h$ at the origin is the convex hull in $\mathbb{R}_{\geq 0}^{d}$ of $\bigcup_{k \in \operatorname{supp} h} \underline{k}+\mathbb{R}_{\geq 0}^{d}$ and the Newton polytope of $h$ at the origin is the compact boundary of the Newton polyhedron of $h$ at the origin.

Let $\nu_{1}, \ldots, \nu_{r}$ be the monomial valuations on $\mathcal{O}_{\mathbb{C}^{d}, o}$ corresponding to the facets of the Newton polytope of $h$, i.e. for a compact facet $\tau$ with the affine space through $\tau$ given by the equation $a_{1} x_{1}+\cdots+a_{d} x_{d}=N_{\tau}$, the corresponding valuation $\nu$ acts as follows: $\nu\left(x_{1}^{m_{1}} \ldots x_{d}^{m_{d}}\right)=a_{1} m_{1}+\cdots+a_{d} m_{d}$. Ebeling and Gusein-Zade study the Poincaré series $P_{\left\{\omega_{i}\right\}}(\underline{t})$ on $V$ induced by the order functions

$$
\omega_{i}(g):=\max \left\{\nu_{i}\left(g^{\prime}\right) \mid g^{\prime}-g \in(h)\right\} .
$$

On the other hand, we can consider the Poincaré series as defined in Definition 1.1, with $\mathcal{I}=(h)$. The Poincaré series in [E,G-Z2] has a more geometric meaning because one has fibres $F_{\underline{v}}$ and $P_{\left\{\omega_{i}\right\}}(\underline{t})=\sum_{\underline{v} \in \mathbb{Z}^{r}} \chi\left(\mathbb{P} F_{\underline{v}}\right) \underline{t} \underline{v}$. However, our Poincaré series which is rather algebraic - contains more information about the singularity $(V, o)$. In general, the embedded filtration we introduce in this article is richer because $I(\underline{v})$ is not necessarily determined by $I\left(v_{1}, 0, \ldots, 0\right), I\left(0, v_{2}, 0, \ldots, 0\right), \ldots, I\left(0, \ldots, 0, v_{d}\right)$, whereas the ideals that appear in the Poincaré series $P_{\left\{\omega_{i}\right\}}(\underline{t})$ are. We comment further on this in this section.

In [E,G-Z2] a Newton polytope is called stellar if all its facets have a common vertex. If the Newton polytope of $h$ is stellar, then they show [E,G-Z2, Theorem 2] that

$$
P_{\left\{\omega_{i}\right\}}(\underline{t})=\left(1-\underline{t}^{\underline{\nu}(h)}\right) P \underline{\nu}_{X}(\underline{t}) .
$$


However, if the Newton polytope is not stellar, the information on $(V, o)$ can be lost in the Poincaré series $P_{\left\{\omega_{i}\right\}}(\underline{t})$. Indeed, for $h$ a germ of a holomorphic function on $\left(\mathbb{C}^{2}, o\right)$ with $o$ an isolated critical point of $h$, they prove [E,G-Z2, Theorem 1$]$ that

$$
P_{\left\{\omega_{i}\right\}}(\underline{t})=P_{X}^{\underline{\nu}}(\underline{t}) .
$$

Proposition 2.1. If the ideals $I(\underline{v})$ satisfy Condition (1.1), then $P_{\left\{\omega_{i}\right\}}(\underline{t})=\mathcal{P} \frac{\nu}{V}(\underline{t})$.

Proof. It is sufficient to verify that then

$$
I\left(0, \ldots, 0, v_{i}, 0, \ldots, 0\right)=\left\{g \in \mathcal{O}_{V, o} \mid \omega_{i}(g) \geq v_{i}\right\}
$$

We will now characterize the germs $h$ of holomorphic functions on $\mathbb{C}^{d}$ for which Condition (1.1) is satisfied, and thus for which these geometric and algebraic Poincaré series coincide. Obviously this will depend on the Newton polytope of $h$. We will use the following lemma to give the characterization.

Lemma 2.1. Let $\{M(\underline{v})\}$ be ideals in a local ring $R$ that satisfy Condition (1.1) and consider an ideal $\mathcal{I}$ in $R$. Then for $\underline{v_{1}}, \underline{v_{2}} \in \mathbb{Z}^{r}$, the following conditions are equivalent:

(1) $\left(M\left(\underline{v_{1}}\right)+\mathcal{I}\right) \cap\left(M\left(v_{2}\right)+\mathcal{I}\right)=M(\underline{v})+\mathcal{I}$ where $\underline{v}$ is the tuple of the componentwise maxima of $v_{1}$ and $\underline{v_{2}}$;

(2) $\left.\left(M\left(\underline{v_{1}}\right)+\overline{M(} \underline{v_{2}}\right)\right) \overline{\cap \mathcal{I}}=\left(M\left(\underline{v_{1}}\right) \cap \mathcal{I}\right)+\left(M\left(\underline{v_{2}}\right) \cap \mathcal{I}\right)$.

Proof. Suppose that the first equation of sets holds. Take $f \in\left(M\left(\underline{v_{1}}\right)+M\left(\underline{v_{2}}\right)\right) \cap \mathcal{I}$. Then $f=f_{1}+f_{2}$ with $f \in \mathcal{I}, f_{1} \in M\left(\underline{v_{1}}\right)$ and $f_{2} \in M\left(\underline{v_{2}}\right)$. Thus $f_{1} \in\left(M\left(\underline{v_{1}}\right)+\mathcal{I}\right) \cap$

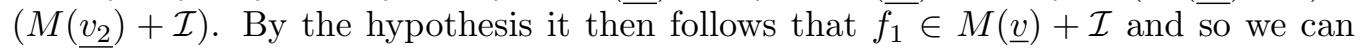
write $f_{1}=g+k$ with $g \in M(\underline{v})$ and $k \in \mathcal{I}$. We have $f=\left(f_{1}-g\right)+\left(f_{2}+g\right)$ and $f_{1}-g \in M\left(\underline{v_{1}}\right) \cap \mathcal{I}$ and $f_{2}+g \in M\left(\underline{v_{2}}\right) \cap \mathcal{I}$. Hence $f \in\left(M\left(\underline{v_{1}}\right) \cap \mathcal{I}\right)+\left(M\left(\underline{v_{2}}\right) \cap \mathcal{I}\right)$.

We now suppose that the second equation of sets holds. We consider the following exact sequences:

$$
\begin{aligned}
0 & \rightarrow\left(M\left(\underline{v_{1}}\right) \cap \mathcal{I}\right) /(M(\underline{v}) \cap \mathcal{I}) \rightarrow M\left(\underline{v_{1}}\right) / M(\underline{v}) \rightarrow\left(M\left(\underline{v_{1}}\right)+\mathcal{I}\right) /(M(\underline{v})+\mathcal{I}) \rightarrow 0 \\
0 & \rightarrow\left(M\left(\underline{v_{2}}\right) \cap \mathcal{I}\right) /(M(\underline{v}) \cap \mathcal{I}) \rightarrow M\left(\underline{v_{2}}\right) / M(\underline{v}) \rightarrow\left(M\left(\underline{v_{2}}\right)+\mathcal{I}\right) /(M(\underline{v})+\mathcal{I}) \rightarrow 0 .
\end{aligned}
$$

We take a set $B_{i}^{\prime}$ of elements in $M\left(v_{i}\right) \cap \mathcal{I}$ that give rise to a basis of the vector space $\left(M\left(v_{i}\right) \cap \mathcal{I}\right) /(M(\underline{v}) \cap \mathcal{I}), i \in\{1,2\}$. For $i \in\{1,2\}$, we use the exact sequences (2.1) and (2.2) to add to $B_{i}^{\prime}$ a set of elements $B_{i}^{\prime \prime}$ of $M\left(\underline{v_{i}}\right)$ whose images are a basis of the quotient $\left(M\left(\underline{v_{i}}\right)+\mathcal{I}\right) /(M(\underline{v})+\mathcal{I})$. So the classes of the elements of $B_{i}:=B_{i}^{\prime} \cup B_{i}^{\prime \prime}$ form a basis of $M\left(v_{i}\right) / M(\underline{v}), i=1,2$. We also have that the classes of the elements in $B_{1} \cup B_{2}$ form a system of generators for the vector space $\left(M\left(\underline{v_{1}}\right)+M\left(\underline{v_{2}}\right)\right) / M(\underline{v})$. Analogously, the classes of the elements in $B_{1}^{\prime} \cup B_{2}^{\prime}$ are a system of generators for the vector space $\left[\left(M\left(\underline{v_{1}}\right) \cap \mathcal{I}\right)+\left(M\left(\underline{v_{2}}\right) \cap \mathcal{I}\right)\right] /(M(\underline{v}) \cap \mathcal{I})$.

Condition (1.1) implies that the set $B_{1} \cup B_{2}$ consists of elements of $M\left(\underline{v_{1}}\right)+M\left(\underline{v_{2}}\right)$ whose classes module $M(\underline{v})$ are linearly independent and hence the classes of the elements in $B_{1} \cup B_{2}$ are a basis of $\left(M\left(\underline{v_{1}}\right)+M\left(\underline{v_{2}}\right)\right) / M(\underline{v})$. Indeed, if $x$ is a linear combination of elements in $B_{1}$ and $y$ is a linear combination of elements of $B_{2}$ and 
if $x+y=z$ with $z \in M(\underline{v})$, then $x=z-y \in M\left(\underline{v_{1}}\right) \cap M\left(\underline{v_{2}}\right)$. Condition (1.1) then implies that $x, y \in M(\underline{v})$.

Let us now take an element $f \in\left(M\left(v_{1}\right)+\mathcal{I}\right) \cap\left(M\left(v_{2}\right)+\mathcal{I}\right)$, so $f=f_{1}+k_{1}=f_{2}+k_{2}$, $f_{i} \in M\left(\underline{v_{i}}\right)$ and $k_{i} \in \mathcal{I}, i=1,2$. For $i=1,2$, we can write $f_{i}=f_{i}^{\prime}+f_{i}^{\prime \prime}+m_{i}$ with $f_{i}^{\prime}$ a linear combination of elements in $B_{i}^{\prime}, f_{i}^{\prime \prime}$ a linear combination of elements in $B_{i}^{\prime \prime}$ and $m_{i} \in M(\underline{v})$. Then $g:=k_{2}-k_{1}=f_{1}-f_{2} \in\left(M\left(\underline{v_{1}}\right)+M\left(\underline{v_{2}}\right)\right) \cap \mathcal{I}=$ $\left(M\left(\underline{v_{1}}\right) \cap \mathcal{I}\right)+\left(M\left(\underline{v_{2}}\right) \cap \mathcal{I}\right)$ by the hypothesis. Hence $g=g_{1}^{\prime}+g_{2}^{\prime}+m$, with $g_{i}^{\prime}$ a linear combination of elements of $B_{i}^{\prime}, i=1,2$, and $m \in M(\underline{v}) \cap \mathcal{I}$. We obtain

$$
\left(g_{1}^{\prime}-f_{1}^{\prime}\right)-f_{1}^{\prime \prime}+\left(g_{2}^{\prime}+f_{2}^{\prime}\right)+f_{2}^{\prime \prime}=m_{1}-m_{2}-m .
$$

The right-hand side of this equality is contained in $M(\underline{v})$. The left-hand side is a sum of four terms that are linear combinations of elements, respectively, from $B_{1}^{\prime}, B_{1}^{\prime \prime}, B_{2}^{\prime}$ and $B_{2}^{\prime \prime}$. As the classes of the elements in $B_{1} \cup B_{2}$ are a basis of $\left(M\left(\underline{v_{1}}\right)+M\left(\underline{v_{2}}\right)\right) / M(\underline{v})$, we get in particular $f_{1}^{\prime \prime}=0$. Hence $f=m_{1}+\left(f_{1}^{\prime}+k_{1}\right) \in M(\underline{v})+\overline{\mathcal{I}}$.

Definition 2.1. A Newton polytope is called bi-stellar if every two facets of the Newton polytope have a nonempty intersection.

Proposition 2.2. The Newton polytope of $h$ is bi-stellar if and only if the ideals $M(\underline{v})+(h)$ satisfy Condition (1.1).

Proof. Say the Newton polytope of $h$ has $r$ compact facets inducing the monomial valuations $\nu_{1}, \ldots, \nu_{r}$ on $\mathbb{C}^{d}$. Suppose that the Newton polytope of $h$ is bi-stellar. By Lemma 2.1, it suffices to show that for all $\underline{v_{1}}, \underline{v_{2}} \in \mathbb{Z}^{r}$ one has that

$$
\left(I\left(\underline{v_{1}}\right) \cap(h)\right)+\left(I\left(\underline{v_{2}}\right) \cap(h)\right)=\left(I\left(\underline{v_{1}}\right)+I\left(\underline{v_{2}}\right)\right) \cap(h) .
$$

Let $\left.g h \in I\left(\underline{v_{1}}\right)+I\left(\underline{v_{2}}\right)\right)$ and $\underline{q}:=\underline{\nu}(h)$. We write $g=g_{1}+g_{2}$ with $g_{1}=\sum \lambda_{\underline{a}} \underline{x} \underline{\underline{a}}$ and $\underline{x} \underline{\underline{a}} \notin I\left(\underline{v_{1}}-\underline{q}\right)+I\left(\underline{v_{2}}-\underline{\bar{q}}\right)$, for all $\underline{x}^{\underline{a}}$ in supp $g_{1}$, and $g_{2}=\sum \lambda_{\underline{b}} \underline{\underline{x}} \underline{\underline{b}}$ with $\underline{x} \underline{\underline{b}} \in$ $I\left(\underline{v_{1}}-\underline{q}\right)+I\left(\underline{v_{2}}-\underline{q}\right)$, for all $\underline{x}^{\underline{b}}$ in supp $g_{2}$. Suppose that $g_{1} \neq 0$. We take a monomial $\underline{x}^{\underline{a}}$ in supp $g_{1}$. Then there exist $i, j \in\{1, \ldots, r\}$ such that $\nu_{i}\left(\underline{x}^{\underline{a}}\right)<v_{1, i}-q_{i}$ and $\nu_{j}\left(\underline{x}^{\underline{a}}\right)<v_{2, j}-q_{j}$.

We first consider the case where $i \neq j$. Let $N$ be the set of the monomials in supp $g_{1}$ that are minimal for the pair $\left(\nu_{i}, \nu_{j}\right)$, i.e. $\underline{x}^{\underline{c}} \in N$ if and only if there does not exist a monomial $\underline{x}^{\underline{d}}$ in supp $g_{1}$ for which $\nu_{i}\left(\underline{x}^{\underline{d}}\right)<\nu_{i}\left(\underline{x}^{\underline{c}}\right)$ and $\nu_{j}\left(\underline{x}^{\underline{d}}\right)<\nu_{j}\left(\underline{x}^{\underline{c}}\right)$. Let $M$ be the set of monomials $\underline{x}^{\underline{m}}$ in supp $h$ for which $\nu_{i}\left(\underline{x}^{\underline{m}}\right)=q_{i}$ and $\nu_{j}\left(\underline{x}^{\underline{m}}\right)=q_{j}$. As the Newton polytope is bi-stellar, $M$ is not empty. For the monomials $\underline{x}^{\underline{m}} \underline{x}^{\underline{\underline{c}}}$ with $\underline{x} \underline{\underline{m}} \in M$ and $\underline{x} \underline{\underline{c}} \in N$, we thus have that $\nu_{i}(\underline{x} \underline{\underline{m}} \underline{x} \underline{\underline{c}})<v_{1, i}$ and $\nu_{j}(\underline{x} \underline{\underline{m}} \underline{x} \underline{\underline{c}})<v_{2, j}$. As such monomials do not appear in the support of $g h$ they should be cancelled. It follows that at least one such monomial $\underline{x}^{\underline{m}} \underline{x}^{\underline{c}}$ has to be equal to a monomial $\underline{x}^{\underline{h}} \underline{x}^{\underline{a}^{\prime}}$ with $\underline{x}^{\underline{h}} \in \operatorname{supp} h \backslash M$ and $\underline{x}^{\underline{a}^{\prime}}$ in supp $g_{1}$. Say $\nu_{i}\left(\underline{x}^{\underline{h}}\right)>q_{i}$. We then find that $\nu_{i}\left(\underline{x}^{a^{\prime}}\right)<\nu_{i}\left(\underline{x}^{\underline{c}}\right)$ and $\nu_{j}\left(\underline{x}^{\underline{a^{\prime}}}\right) \leq \nu_{j}\left(\underline{x}^{\underline{c}}\right)$ which contradicts the fact that $\underline{x}^{\underline{c}} \in N$ and thus $g_{1}=0$.

Suppose now that $i=j$. Let $N$ be the set of monomials with support in $g_{1}$ that are minimal for the valuation $\nu_{i}$, i.e. $x^{\underline{c}} \in N$ if and only if there does not exist a monomial $\underline{x}^{\underline{d}}$ in supp $g_{1}$ for which $\nu_{i}(\underline{\underline{x}} \underline{\underline{d}})<\nu_{i}(\underline{x} \underline{\underline{c}})$. Let $M$ be the set of monomials $\underline{x}^{\underline{m}}$ in the support of $h$ for which $\nu_{i}\left(\underline{x}^{\underline{m}}\right)=q_{i}$. Analogously, there then has to be a monomial $\underline{x}^{\underline{m}} \underline{x}^{\underline{c}}$ with $\underline{x}^{\underline{m}} \in M$ and $\underline{x}^{\underline{c}} \in N$ that is equal to a monomial $\underline{x}^{\underline{h}} \underline{x}^{\underline{a}^{\prime}}$, with 
$\underline{x}^{\underline{h}} \in \operatorname{supp} h \backslash M$ and $\underline{x}^{\underline{a^{\prime}}} \in \operatorname{supp} g_{1}$. Again we get a contradiction because $\underline{x}^{\underline{c}}$ would not be minimal for $\nu_{i}$.

We now suppose that the ideals $M(\underline{v})+(h)$ satisfy Condition (1.1). If the Newton polytope of $h$ would not be bi-stellar, then there would exist two valuations $\nu_{i}, \nu_{j}$ with $i, j \in\{1, \ldots, r\}$ for which the sets of monomials $M_{i}=\left\{\underline{x}^{\underline{m}} \in \operatorname{supp}(h) \mid \nu_{i}\left(\underline{x}^{\underline{m}}\right)=q_{i}\right\}$ and $M_{j}=\left\{\underline{x} \underline{\underline{m}} \in \operatorname{supp}(h) \mid \nu_{j}(\underline{x} \underline{\underline{m}})=q_{j}\right\}$ would be disjoint. Let $h_{i}$ be the part of $h$ with support in $M_{i}$, so $\nu_{i}\left(h_{i}\right)=q_{i}$ and $\nu_{i}\left(h-h_{i}\right)>q_{i}$. Then $h=h_{i}+\left(h-h_{i}\right)$ with $\nu_{i}\left(h_{i}\right)<\nu_{i}\left(h-h_{i}\right), \nu_{j}\left(h-h_{i}\right)<\nu_{j}\left(h_{i}\right)$ and $\underline{\nu}(h) \leq \underline{\nu}\left(h_{i}\right)$ and $\underline{\nu}(h) \leq \underline{\nu}\left(h-h_{i}\right)$. By Lemma 2.1 it follows that $h$ would be contained in $\left(M\left(\underline{\nu}\left(h_{i}\right)\right)+M\left(\underline{\nu}\left(h-h_{i}\right)\right)\right) \cap(h)$ but not in $\left.\left(M\left(\underline{\nu}\left(h_{i}\right)\right) \cap(h)\right)+\left(M\left(\underline{\nu}\left(h-h_{i}\right)\right)\right) \cap(h)\right)$, contradicting Condition (1.1).

\section{Embedded filtrations for plane curve singularities}

Let $X=\mathbb{C}^{2}$ and let $\mathcal{I}$ be a primary ideal in $\mathcal{O}_{X, o}$. If $\phi: Z \rightarrow \mathbb{C}^{2}$ is a principilization of $\mathcal{I}$, then $\phi$ is realized by blowing up a constellation of points $\left\{Q_{\sigma}\right\}_{\sigma \in G}$. The map $\phi$ factorizes through the normalized blowing up of $\mathcal{I}$ which we will denote by $\overline{B l_{\mathcal{I}}\left(\mathbb{C}^{2}\right)}$. Let $\sigma$ be the morphism $Z \rightarrow \overline{B l_{\mathcal{I}}\left(\mathbb{C}^{2}\right)}$ in this factorization. For $\sigma \in G$, we denote the exceptional divisor of the blowing-up in $Q_{\sigma}$ by $E_{\sigma}$, as well as its strict transform under following blowing-ups, and $\mathcal{D}:=\cup_{\sigma \in G} E_{\sigma}$. Blowing up a point $Q_{\sigma}$ induces a discrete valuation $\nu_{\sigma}$ on $\mathbb{C}(X) \backslash\{0\}$ : for $g \in \mathbb{C}(X) \backslash\{0\}$, the value $\nu_{\sigma}(g)$ is the order of the pullback of $\mathrm{g}$ along $E_{\sigma}$. The valuation $\nu_{\sigma}$ is called Rees for $\mathcal{I}$ if its centre in $\overline{B l_{\mathcal{I}}(X)}$ is a divisor. We have that $\nu_{\sigma}$ is Rees for $\mathcal{I}$ if and only if the strict transform of the curve given by a general element in $\mathcal{I}$ intersects $E_{\sigma}$ (see for example [L,VP, Lemma 8]). Say that $E_{1}, \ldots, E_{r}$ are the exceptional components that give rise to Rees valuations $\nu_{1}, \ldots, \nu_{r}$.

Let us now consider a general element $h$ in the ideal $\mathcal{I}$ and let $V$ be the hypersurface given by $\{h=0\}$. In [C,D,G-Z2], one studied the Poincaré series $P_{V}^{\underline{\nu}}(\underline{t})$ that is defined by the filtration on $\mathcal{O}_{V, o}$ induced by the essential valuations $\underline{\nu}$ of the minimal resolution of the plane curve $V$. One showed that Poincaré series contained the same information as the embedded topology of the curve and that $P_{V}^{\nu}(t, \ldots, t)$ equals the zeta function of monodromy.

We will now study the Poincaré series of the embedded filtration on $\mathcal{O}_{V, o}$ induced by the Rees valuations $\underline{\nu}=\left(\nu_{1}, \ldots, \nu_{r}\right)$. For $1 \leq j \leq r$, suppose that $E_{j}$ is intersected $n_{j}$ times by the strict transform of $\{h=0\}$. Let $E_{\sigma}^{\bullet}$ be $E_{\sigma}$ without the intersection points with the other components of $\mathcal{D}$ and let $E_{\sigma}^{\circ}$ be $E_{\sigma}$ without the intersection points of the other components of $\phi^{-1}\left(h^{-1}\{o\}\right)$. Let $I$ be the intersection matrix of the $\left\{E_{\sigma}\right\}_{\sigma \in G}$ and let $M=-I^{-1}$. Let $\mathcal{C}_{\sigma}$ be a curvette through $E_{\sigma}$ (i.e., the projection by $\phi$ of a smooth curve transversal to $E_{\sigma}$ and not intersecting other components of $\mathcal{D})$. The entry $m_{\sigma, \tau}$ in $M$ is then also equal to $\nu_{\tau}\left(\mathcal{C}_{\sigma}\right)$.

Theorem 3.1. The Poincaré series $\mathcal{P} \frac{\nu}{V}(\underline{t})$ determines and is determined by the embedded topology of $\{h=0\}$.

Proof. By Theorem 1.1, $\mathcal{P} \frac{\nu}{V}\left(t_{1}, \ldots, t_{r}\right)=\left(1-t_{1}^{q_{1}} \cdots t_{r}^{q_{r}}\right) P_{X}^{\nu}\left(t_{1}, \ldots, t_{r}\right)$, with $\underline{q}=\underline{\nu}(h)$. The Poincaré series $P{ }_{\bar{X}}(\underline{\underline{t}})$ induced by plane divisorial valuations is computed in $[\mathrm{D}, \mathrm{G}-\mathrm{Z}]$. For $\sigma \in G$, let $\underline{m_{\sigma}}=\left(m_{\sigma, 1}, \ldots, m_{\sigma, r}\right)$. Then one has

$$
P \frac{\nu}{X}\left(t_{1}, \ldots, t_{r}\right)=\prod_{\sigma \in G}\left(1-\underline{t}^{\underline{m}^{\sigma}}\right)^{-\chi\left(E_{\sigma}^{\bullet}\right)} .
$$


As $E_{j}$ is intersected $n_{j}$ times by the strict transform of $\{h=0\}, 1 \leq j \leq r$, we get $q_{i}=\sum_{j=1}^{r} n_{j} m_{i, j}$, for $1 \leq i \leq r$. If the curve is irreducible (i.e. $r=1$ ) and if $E_{1}$ is intersected by the strict transform then $n_{1}=1$ and $q_{1}=m_{1,1}$. We then have

$$
\mathcal{P} \frac{\nu}{V}(t)=\left(1-t^{m_{1,1}}\right) \prod_{\sigma \in G}\left(1-t^{m_{\sigma, 1}}\right)^{-\chi\left(E_{\sigma}^{\bullet}\right)}=\prod_{\sigma \in G}\left(1-t^{q_{\sigma}}\right)^{-\chi\left(E_{\sigma}^{\circ}\right)} .
$$

Hence, it follows that $\mathcal{P} \frac{\nu}{V}(t)$ is then equal to the zeta function of monodromy $\zeta_{V}(t)$ $\left[\mathrm{A}^{\prime} \mathrm{C}\right]$.

Suppose now that the curve is reducible. We will show that the factor $(1-\underline{t} \underline{q})$ can not be cancelled by a factor $\left(1-\underline{t}^{\sigma}\right)^{-\chi\left(E_{\sigma}^{*}\right)}$ of $P_{\bar{X}}^{\underline{\nu}}(\underline{t})$. As $q_{i}=\sum_{j=1}^{r} n_{j} m_{i, j}$, we have that $q_{i}>m_{i, j}$ for all $j \in\{1, \ldots, r\}$. If $\nu_{\sigma}$ is not a Rees valuation, then there exists always a valuation $\nu_{j}$ which is Rees and such that $Q_{j}$ lies above $Q_{\sigma}$. Then $m_{\sigma, j}<m_{j, j}$ and thus $q_{i}>m_{\sigma, i}$, for all $\sigma \in G$. Thus $\underline{q}$ is the biggest exponent in the cyclotomic factors in $\mathcal{P} \frac{\nu}{V}(\underline{t})$. This makes that we can extract the value $\underline{q}$ and the Poincaré series $P_{\bar{X}}^{\nu}(\underline{t})$ from the Poincaré series $\mathcal{P} \underline{\nu}(\underline{t})$. In [C,D,G-Z4], it has been shown that $P_{\bar{X}}^{\nu}(\underline{t})$ determines the dual graph of the divisors $\left\{E_{\sigma}\right\}_{\sigma \in G}$ and thus the matrix $M$. As $M$ is invertible, it follows that we can now compute the numbers $n_{j}, 1 \leq j \leq r$. Hence the dual resolution graph of $\{h=0\}$ is known and the Poincaré series $\mathcal{P} \frac{\nu}{V}(\underline{t})$ determines the embedded topology of $\{h=0\}$.

For the Poincaré series we study here, we get that the zeta function of monodromy

$$
\zeta_{V}(t)=\frac{\mathcal{P} \frac{\nu}{V}\left(t^{n_{1}}, \ldots, t^{n_{r}}\right) \prod_{j=1}^{r}\left(1-t^{q_{j}}\right)^{n_{j}}}{\left(1-t^{\sum_{j=1}^{r} n_{j} q_{j}}\right)} .
$$

Remark 3.1. If $V=\{h=0\}$ is a reduced plane curve singularity and $Z \rightarrow \mathbb{C}^{2}$ is a concrete embedded resolution of singularities for $V$, then the function $h$ becomes a general element for some convenient primary ideal $\mathcal{I}$ such that $\mathcal{I} \mathcal{O}_{Z}$ is locally principal. Hence Theorem 3.1 can be applied to any reduced curve singularity and a chosen embedded resolution for it and, in particular, for the minimal one.

\section{Poincaré series for toric varieties}

We now consider the particular case where $V$ is an affine toric variety. Let $S$ be a semigroup in $M \cong \mathbb{Z}^{d}$ such that $S+(-S)=M$ and $S \cap(-S)=0$ and let $V=$ Spec $\mathbb{C}[S]$ be the associated affine toric variety. Let $\check{\sigma}$ be the cone generated by $S$ and let $\left\{s_{1}, \ldots, s_{d+p}\right\}$ be a system of generators of $S=\check{\sigma} \cap M$. Suppose that the embedding of $V$ in $\mathbb{C}^{d+p}$ is given by the map

$$
\begin{aligned}
\varepsilon: \mathbb{C}\left[x_{1}, \ldots, x_{d+p}\right] & \rightarrow \mathbb{C}[S] \\
x_{k} & \mapsto \chi^{s_{k}} .
\end{aligned}
$$

We set $\operatorname{deg}\left(x_{k}\right)=s_{k}, 1 \leq k \leq d+p$. The toric variety $V$ is given by an ideal generated by binomials $h^{i}=\underline{x} \underline{\underline{\alpha}^{i}}-\underline{x} \underline{\beta^{i}} \in \mathbb{C}\left[x_{1}, \ldots, x_{d+p}\right], 1 \leq i \leq p$. Moreover $\operatorname{deg}\left(\underline{x} \underline{\alpha}^{i}\right)=\operatorname{deg}\left(\underline{x} \underline{\beta}^{i}\right)$ and $\operatorname{supp}\left(\underline{x} \underline{\alpha}^{\alpha^{i}}\right) \cap \operatorname{supp}\left(\underline{x}{\underline{\beta^{i}}}^{i}\right)=\emptyset$. The Newton polytope of each toric hypersurface $h^{i}$ is a segment $\tau^{i}$, connecting $\underline{\alpha}^{i}$ and $\beta^{i}$. Let $\tau^{* i}$ be the dual space in $\mathbb{R}_{\geq 0}^{* d+p}$ to this segment and let $H^{* i}$ be the hyperplane passing through $\tau^{* i}$. The equation of $H^{* i}$ is $\sum_{k=1}^{d+p}\left(\alpha_{k}^{i}-\beta_{k}^{i}\right) x_{k}=0$. Set $\tau^{*}:=\tau^{* 1} \cap \cdots \cap \tau^{* p}$ and $H^{*}:=$ $H^{* 1} \cap \cdots \cap H^{* p}$. Let $N$ be the dual space to $M$ and let $\sigma$ be the dual cone to $\check{\sigma}$. 
A primitive element $n$ in $\sigma \cap N$ defines a discrete valuation $\nu$ of $\mathbb{C}(V)$ by setting $\nu\left(\sum_{m \in F} a_{m} \chi^{m}\right)=\min \left\{\langle m, n\rangle \mid m \in F, a_{m} \neq 0\right\}$.

A finite set of valuations $\underline{\nu}$ in $\sigma$ induces a Poincaré series $P_{V}^{\underline{\nu}}$ for $V$. On the other hand, valuations $\mu$ in $\tau^{*}$ give rise to ambient ideals $M(\underline{v}) \subset X=\mathbb{C}^{d+p}$ and hence to a Poincaré series $\overline{\mathcal{P}} \frac{\mu}{V}$ for $V$. We now show how both Poincaré series are related.

Theorem 4.1. The cones $\sigma$ and $\tau^{*}$ are isomorphic and under this isomorphism one has $P_{V}^{\underline{\nu}}(\underline{t})=\mathcal{P} \frac{\mu}{V}(\underline{t})$.

Proof. We show that there is an isomorphism $\theta: \mathbb{R}^{d} \rightarrow H^{*}$ that maps $\sigma$ to $\tau^{*}$. Let $\nu \in \mathbb{R}^{d}$ and let $\mu=\left(\mu^{1}, \ldots, \mu^{d+p}\right)$ be the vector such that $\mu^{k}=\left\langle s_{k}, \nu\right\rangle, 1 \leq k \leq d+p$. Then obviously $\sum_{k=1}^{d+p}\left(\alpha_{k}^{i}-\beta_{k}^{i}\right) \mu^{k}=0$ for $1 \leq i \leq p$ and this implies that $\mu \in H^{*}$. For the opposite direction, take $\mu \in H^{*}$. The equality $\sum_{k=1}^{d+p}\left(\alpha_{k}^{i}-\beta_{k}^{i}\right) \mu^{k}=0$ implies that there exists a $\nu \in \mathbb{R}^{d}$ such that $\mu^{k}=\left\langle s_{k}, \nu\right\rangle, 1 \leq k \leq d+p$. This $\nu$ is then unique. As $\sigma$ is the dual cone to $\check{\sigma}$, it follows that $\sigma$ maps to $\tau^{*}$.

The Poincaré series $\mathcal{P} \frac{\mu}{V}$ with respect to the valuations $\mu_{1}, \ldots, \mu_{r} \in \tau^{*}$ is induced by the ideals

$$
J(\underline{v})=\left(\underline{x}^{\lambda} \mid\left\langle\lambda, \mu_{j}\right\rangle \geq v_{j}, 1 \leq j \leq r\right)+\left(h^{1}, \ldots, h^{p}\right) .
$$

As $\left\langle\lambda, \mu_{j}\right\rangle=\left\langle s, \nu_{j}\right\rangle$, where $s=\sum_{k=1}^{d+p} \lambda_{k} s_{k}$, it follows that $\underline{x}^{\lambda} \in J(\underline{v})$ if and only if $\varepsilon\left(\underline{x}^{\lambda}\right)=\chi^{s}$, with $\left\langle s, \nu_{j}\right\rangle \geq v_{j}, 1 \leq j \leq r$.

The Poincaré series $P_{V}^{\underline{\nu}}$ with respect to the corresponding valuations $\nu_{j}$ is induced by the ideals

$$
\left(\chi^{s} \mid\left\langle s, \nu_{j}\right\rangle \geq v_{j}, 1 \leq j \leq r\right)
$$

It now follows that both Poincaré series coincide.

\section{Embedded filtrations for nondegenerate singularities}

Let $h$ be the germ of a holomorphic function on $\mathbb{C}^{d}$ defining a hypersurface singularity $(V, o)$. Let $\underline{\nu}=\left\{\nu_{1}, \ldots, \nu_{r}\right\}$ be the monomial valuations corresponding to the facets of the Newton polyhedron of $h$, including the noncompact facets. The centre of the valuations $\nu_{i}$ could then be a prime ideal different from the maximal ideal of $\mathcal{O}_{\mathbb{C}^{d}, o}$. We suppose that there is at least one compact facet such that there is at least one valuation with centre in the maximal ideal. The definition of Poincaré series for affine toric varieties can be extended for a set of valuations which contains at least one valuation with centre in the maximal ideal. Indeed, notice that the $\chi\left(\mathbb{P} F_{v}\right)$ are then finite numbers (see also [GP,H] for an equivalent definition using graded rings) such that the Poincaré series $\sum_{\underline{v} \in \mathbb{Z}^{r}} \chi\left(\mathbb{P} F_{\underline{v}}\right) \underline{t} \underline{v}$ is well defined.

Theorem 5.1. Suppose that $h$ is nondegenerate with respect to its Newton polyhedron $\mathcal{N}$ in the origin and that $\mathcal{N}$ has at least one compact facet. Let $\underline{\nu}=\left\{\nu_{1}, \ldots, \nu_{r}\right\}$ be the monomial valuations on $\mathbb{C}^{d}$ induced by the facets of $\mathcal{N}$. Then the Poincaré series $\mathcal{P} \frac{\nu}{V}(\underline{t})$ contains the same information as the Newton polyhedron of $h$ and in particular determines the zeta function of monodromy of $h$.

Proof. Let $\nu_{1}$ be a valuation in $\underline{\nu}$ with centre in the maximal ideal. The coefficient of $\underline{t} \underline{v}$ in the series

$$
\frac{\left(1-t_{1}^{q_{1}} \cdots t_{r}^{q_{r}}\right)}{\left(1-t_{1}^{\nu_{1,1}} \cdots t_{r}^{\nu_{r, 1}}\right) \cdots\left(1-t_{1}^{\nu_{1, d}} \cdots t_{r}^{\nu_{r, d}}\right)}
$$


with $\underline{q}=\underline{\nu}(h)$, can also be written as

$$
\begin{aligned}
& \sum_{A \subset\{2, \ldots, r\}}(-1)^{\# A} \operatorname{dim} \frac{M\left(\underline{v}-\underline{1}_{A}+\underline{1}\right)}{M\left(\underline{v}-\underline{1}_{A}+\underline{e}_{1}+\underline{1}\right)} \\
& -\sum_{A \subset\{2, \ldots, r\}}(-1)^{\# A} \operatorname{dim} \frac{M\left(\underline{v}-\underline{q}-\underline{1}_{A}+\underline{1}\right)}{M\left(\underline{v}-\underline{q}-\underline{1}_{A}+\underline{e}_{1}+\underline{1}\right)} .
\end{aligned}
$$

Notice that these dimensions are finite because $\nu_{1}$ has centre in the maximal ideal. One can now argue in the same way as in the proof of Theorem 1.1 to obtain that

$$
\mathcal{P} \frac{\nu}{V}(\underline{t})=(1-\underline{t} \underline{q}) P_{\mathbb{C}^{d}}^{\underline{\nu}}(\underline{t})
$$

We show that no factors cancel in (5.1). Indeed, from the fact that $\underline{\nu}$ contains the valuations $(1,0, \ldots, 0), \ldots,(0, \ldots, 0,1)$ and at least one valuation where none of the entries is equal to 0 , one can deduce that some variable, let us say $x_{1}$, should divide $h$ and that the monomial $x_{1}$ should be contained in the support of $h$. This contradicts the hypothesis that the Newton polyhedron of $h$ contains at least one compact facet. Picturing the hyperplanes

$$
\nu_{j, 1} x_{1}+\cdots+\nu_{j, d} x_{d}=q_{j}, \quad 1 \leq j \leq r,
$$

the Newton polyhedron of $h$ is completely determined. In particular, for nondegenerate polynomials, one can compute the zeta function of monodromy from the Newton polyhedron by the formula of Varchenko (see [Va]).

\section{Acknowledgment}

The research was partially supported by the Fund of Scientific Research - Flanders and MEC PN I+D+I MTM2007-64704.

\section{References}

[A'C] N. A'Campo, La fonction zêta d'une monodromie, Comment. Math. Helv. 50 (1975), $233-248$.

[C,D,G-Z1] A. Campillo, F. Delgado and S. M. Gusein-Zade, On the monodromy of a plane curve singularity and the Poincaré series of its ring of functions, Funct. Anal. Appl. 33 (1999), $56-57$.

[C,D,G-Z2] A. Campillo, F. Delgado and S. M. Gusein-Zade, The Alexander polynomial of a plane curve singularity via the ring of functions of it, Duke Math. J. 117 (2003), 125-156.

[C,D,G-Z3] A. Campillo, F. Delgado and S. M. Gusein-Zade, Poincaré series of a rational surface singularity, Inv. Math. 155 (2004), 41-53.

[C,D,G-Z4] A. Campillo, F. Delgado and S. M. Gusein-Zade, The Poincaré series of divisorial valuations in the plane defines the topology of the set of divisors, Funct. Anal. Other Math. 3 (2010), 39-46, DOI 10.1007/s11853-010-0040-9.

$[\mathrm{C}, \mathrm{D}, \mathrm{K}] \quad$ A. Campillo, F. Delgado and K. Kiyek, Gorenstein property and symmetry for onedimensional local Cohen-Macaulay rings, Manuscripta Math. 83 (1994), 405-423.

$[\mathrm{C}, \mathrm{H}, \mathrm{R}] \quad$ S. D. Cutkosky, J. Herzog and A. Reguera, Poincaré series of resolutions of surface singularities, Trans. Amer. Math. Soc. 356 (2004), 1833-1874.

[D,G-Z] F. Delgado and S. M. Gusein-Zade, Poincaré series for several plane divisorial valuations, Proc. Edinburgh Math. Soc. 46 (2003), 501-509.

[E,G-Z1] W. Ebeling and S. M. Gusein-Zade, Monodromies and Poincaré series of quasihomogeneous complete intersections, Abh. Math. Sem. Univ. Hamburg 74 (2004), 175-179.

[E,G-Z2] W. Ebeling and S. M. Gusein-Zade, Multi-variable Poincaré series associated with Newton diagrams, J. Singularities 1 (2010), 60-68. 
[GP,H] P. D. González Pérez and F. Hernando, Quasi ordinary singularities, essential divisors and Poincaré series, J. London Math. Soc. (2009), DOI 10.1112/jlms/jdp014.

[L] A. Lemahieu, Poincaré series of a toric variety, J. Algebra 315 (2007), 683-697.

$[\mathrm{L}, \mathrm{VP}] \quad$ A. Lemahieu and L. Van Proeyen, The holomorphy conjecture for ideals in dimension two, Proc. Amer. Math. Soc. 139 (2011), 3845-3852.

[N] A. Némethi, Poincaré series associated with surface singularities, in 'Singularities I', Contemp. Math. 474, Amer. Math. Soc., Providence, RI, 2008, 271-297.

[Va] A. N. Varchenko, Zeta-function of monodromy and Newton's diagram, Inv. Math. 37 (1976), 253-262.

UFR de mathématiques, Université Lille 1, BÂtiment M3, Cité Scientifique, 59655, Villeneuve D'AscQ Cedex, France

E-mail address: lemahieu.ann@gmail.com 
